\title{
Research Article \\ Propagation of Plane Waves in a Thermally Conducting Mixture
}

\author{
Baljeet Singh \\ Department of Mathematics, Post Graduate Government College, Sector 11, Chandigarh 160 011, India \\ Correspondence should be addressed to Baljeet Singh, bsinghgc11@gmail.com
}

Received 10 March 2011; Accepted 4 May 2011

Academic Editors: Y. Dimakopoulos, S. Li, and Y. Liu

Copyright ( 2011 Baljeet Singh. This is an open access article distributed under the Creative Commons Attribution License, which permits unrestricted use, distribution, and reproduction in any medium, provided the original work is properly cited.

The governing equations for generalized thermoelasticity of a mixture of an elastic solid and a Newtonian fluid are formulated in the context of Lord-Shulman and Green-Lindsay theories of generalized thermoelasticity. These equations are solved to show the existence of three coupled longitudinal waves and two coupled transverse waves, which are dispersive in nature. Reflection from a thermally insulated stress-free surface is considered for incidence of coupled longitudinal wave. The speeds and reflection coefficients of plane waves are computed numerically for a particular model.

\section{Introduction}

The soil consists of an assemblage of particles with different sizes and shapes which form a skeleton whose voids are filled by water and air or gas. The word "soil," therefore, implies a mixture of assorted mineral grains with various fluids. The first continuum theory of mixtures was proposed by Truesdell [1] and Truesdell and Toupin [2] in terms of the kinematic and thermodynamic variables associated with each constituent of the mixture. Another theory presented by Green and Naghdi [3] uses the kinematic and thermodynamic variables associated with the mixture as a whole. The classical theory of mixtures was discussed by Atkin and Craine [4] and Bowen [5]. Bedford and Drumheller [6] formulated the theories of immiscible and structured mixtures. Various other theories of mixtures were also developed by Muller [7], Dunwoody [8], Krishnaswamy and Batra [9], Iesan [10-12], Rajagopal and Tao [13], and Pompei and Scalia [14].

Many engineering materials, as well as soils, rocks, granular materials, sand, and underground water mixtures may be modeled more realistically by means of micropolar continua. Eringen [15] developed a theory of micropolar mixture of porous media (nonreacting mixture of micropolar elastic solid and a micropolar viscous fluid at a single temperature) to include the rotational degrees of freedom. Eringen [15] also obtained the 
field equations of a mixture of an elastic solid and a Newtonian fluid as a special case by dropping the micropolar effects. In the present paper, the governing equations for generalized thermoelasticity of mixture of an elastic solid and a Newtonian fluid are formulated in context of Lord-Shulman [16] and Green-Lindsay [17] theories of generalized thermoelasticity. These equations are solved to show the existence of various coupled plane waves. The reflection of these plane waves is studied at thermally insulated stress-free surface and the reflection coefficients are computed and shown graphically with the angle of incidence. Wave propagation in such a media may find its applications in consolidation and oil exploration problems.

\section{Equations of Motion}

We consider a binary mixture of an elastic solid and a Newtonian fluid at the same temperature where no chemical reaction takes place between the two species of the mixture. According to the last section given in Eringen [15], the equations of motion in a linear isotropic thermoelastic mixture of an elastic solid and Newtonian fluid in absence of external loads are written as

$$
\begin{aligned}
& \left(\lambda^{S}+2 \mu^{S}\right) \nabla\left(\nabla \cdot \mathbf{u}^{S}\right)-\mu^{S} \nabla \times\left(\nabla \times \mathbf{u}^{S}\right) \\
& -\xi\left(\dot{\mathbf{u}}^{S}-\dot{\mathbf{u}}^{F}\right)-\left(\frac{S}{T_{0}}+\beta_{0}\right) \nabla\left(T+t_{1} \dot{T}\right)=\rho^{S} \ddot{\mathbf{u}}^{S}, \\
& \left(\lambda^{F}+2 \mu^{F}\right) \nabla\left(\nabla \cdot \dot{\mathbf{u}}^{F}\right)-\mu^{F} \nabla \times\left(\nabla \times \dot{\mathbf{u}}^{F}\right)+\xi\left(\dot{\mathbf{u}}^{S}-\dot{\mathbf{u}}^{F}\right) \\
& \quad+\left(\frac{S}{T_{0}}+\beta_{0}\right) \nabla\left(T+t_{1} \dot{T}\right)=\rho^{F} \ddot{\mathbf{u}}^{F}, \\
& \nabla \cdot\left[S\left(\dot{\mathbf{u}}^{S}-\dot{\mathbf{u}}^{F}\right)+\frac{K^{*}}{T_{0}} \nabla T\right]-\rho\left[\beta_{0} \nabla \cdot\left(\dot{\mathbf{u}}^{S}+t_{0} \Omega \ddot{\mathbf{u}}^{S}\right)+\rho^{F} \delta \nabla \cdot\left(\dot{\mathbf{u}}^{F}+t_{0} \Omega \ddot{\mathbf{u}}^{F}\right)\right] \\
& \quad=\rho C_{0}\left(\dot{T}+t_{0} \ddot{T}\right),
\end{aligned}
$$

where $\mathbf{u}^{S}, \mathbf{u}^{F}$ are displacement vectors in solid and fluid phase, respectively, and $T$ is the temperature variable. The coefficients $\lambda^{S}, \mu^{S}, \beta_{0}$ are the thermoelastic constants for isotropic elastic solids, $\lambda^{F}, \mu^{F}$ are fluid viscosities, $K^{*}$ is classical Fourier constant, $s$ is the heat generation due to velocity difference, $\xi$ is the momentum generation coefficient due to the velocity difference, $C_{0}$ is specific heat at constant deformation, $T_{0}$ is an ambient temperature, $\delta=-\left(\partial \psi / \partial \rho^{F}\right)_{0}, \psi$ is Helmholtz's free energy function, $\rho, \rho^{S}, \rho^{F}$ are the densities of mixture, solid, and fluid in the natural state, superposed dot indicates the temporal derivative, and other symbols have their usual meanings.

The use of symbol $\Omega$, in (2.3), makes these fundamental equations possible for the two different theories of the generalized thermoelasticity. For the L-S (Lord-Shulman) theory $t_{1}=0, \Omega=1$ and for G-L (Green-Lindsay) theory $t_{1}>0$ and $\Omega=0$. The thermal relaxations $t_{0}$ and $t_{1}$ satisfy the inequality $t_{1} \geq t_{0} \geq 0$ for the G-L theory only. 
By introducing the scalar potentials $q^{S}, q^{F}$ and vector potentials $\mathbf{U}^{S}, \mathbf{U}^{F}$ through Helmholtz representation of vector field, we can write

$$
\begin{array}{ll}
\mathbf{u}^{S}=\nabla q^{S}+\nabla \times \mathbf{U}^{S}, & \nabla \cdot \mathbf{U}^{S}=0, \\
\mathbf{u}^{F}=\nabla q^{F}+\nabla \times \mathbf{U}^{F}, & \nabla \cdot \mathbf{U}^{F}=0 .
\end{array}
$$

Using (2.4) into (2.1) to (2.3), we obtain the following five equations, that is, three coupled equations in $q^{S}, q^{F}, T$ and two coupled equations in $\mathbf{U}^{S}, \mathbf{U}^{F}$;

$$
\begin{gathered}
c_{1 S}^{2} \nabla^{2} q^{S}-\bar{\xi}^{S}\left(\dot{q}^{S}-\dot{q}^{F}\right)-{\overline{\beta_{1}}}^{S}\left(T+t_{1} \dot{T}\right)=\ddot{q}^{S}, \\
c_{1 F}^{2} \nabla^{2} \dot{q}^{F}+\bar{\xi}^{F}\left(\dot{q}^{S}-\dot{q}^{F}\right)+{\overline{\beta_{1}}}^{F}\left(T+t_{1} \dot{T}\right)=\ddot{q}^{F}, \\
\overline{K^{*}} \nabla^{2} T+\bar{s} \nabla^{2}\left(\dot{q}^{S}-\dot{q}^{F}\right)-\overline{\beta_{0}}\left(1+t_{0} \Omega \frac{\partial}{\partial t}\right) \nabla^{2} \dot{q}^{S}-\bar{\delta}\left(1+t_{0} \Omega \frac{\partial}{\partial t}\right) \nabla^{2} \dot{q}^{F}=\left(1+t_{0} \frac{\partial}{\partial t}\right) \dot{T}, \\
c_{2 S}^{2} \nabla^{2} \mathbf{U}^{S}-\bar{\xi}^{S}\left(\dot{\mathbf{U}}^{S}-\dot{\mathbf{U}}^{F}\right)=\ddot{\mathbf{U}}^{S}, \\
c_{2 F}^{2} \nabla^{2} \dot{\mathbf{U}}^{F}+\bar{\xi}^{F}\left(\dot{\mathbf{U}}^{S}-\dot{\mathbf{U}}^{F}\right)=\ddot{\mathbf{U}}^{F},
\end{gathered}
$$

where, $c_{1 S}^{2}=\left(\lambda^{S}+2 \mu^{S}\right) / \rho^{S}, c_{2 S}^{2}=\mu^{S} / \rho^{S}, \bar{\xi}^{S}=\xi / \rho^{S},{\overline{\beta_{1}}}^{S}=\beta_{1} / \rho^{S}, c_{1 F}^{2}=\left(\lambda^{F}+2 \mu^{F}\right) / \rho^{F}, c_{2 F}^{2}=$ $\mu^{F} / \rho^{F}, \bar{\xi}^{F}=\xi / \rho^{F},{\overline{\beta_{1}}}^{F}=\beta_{1} / \rho^{F}, \beta_{1}=s / T_{0}+\beta_{0}, \bar{\beta}_{0}=\beta_{0} / C_{0}, \bar{\delta}=\rho \delta / C_{0}, \overline{K^{*}}=K^{*} / \rho C_{0} T_{0}, \bar{s}=$ $s / \rho C_{0}$.

\section{Wave Propagation}

In this section, the plane wave propagation in an infinite thermally conducting mixture of elastic solid and Newtonian fluid is studied. In order to solve (2.5), we consider the following form of plane waves propagating in the positive direction of a unit vector $\mathbf{n}$

$$
\left\{q^{S}, q^{F}, T\right\}=\left\{\overline{q^{S}}, \overline{q^{F}}, \bar{T}\right\} \exp [\iota k(\mathbf{n} \cdot \mathbf{r}-V t)]
$$

where $\overline{q^{S}}, \overline{q^{F}}, \bar{T}$ are the constant complex scalar wave amplitudes, $\iota=\sqrt{-1}, \mathrm{r}$ is the position vector, $V$ is the phase velocity in the direction of $\mathbf{n}, \mathrm{k}$ is the wave number, and $\omega(=k V)$ is angular frequency. Using the values of $q^{S}, q^{F}$, and $T$ from (3.1) into (2.5), we obtain a set of three homogeneous equations in three unknown amplitudes $\overline{q^{S}}, \overline{q^{F}}, \bar{T}$. After eliminating these three unknowns, we get the following cubic equation

$$
\Gamma^{3}+A \Gamma^{2}+B \Gamma+C=0
$$

where $\Gamma=V^{2}$ and the expressions for $A, B, C$ are given in Appendix A. The three roots $V_{1}^{2}, V_{2}^{2}, V_{3}^{2}$ of (3.2) correspond to complex speeds of three dispersive coupled longitudinal 
waves in a thermally conducting mixture of elastic solid and Newtonian fluid for both L-S and G-L theories.

Equation (2.6) can be solved by considering the following form of vector potentials:

$$
\left\{\mathbf{U}^{S}, \mathbf{U}^{F}\right\}=\left\{\mathbf{A}^{S}, \mathbf{A}^{\mathrm{F}}\right\} \exp [\iota k(\mathbf{n} \cdot \mathbf{r}-V t)],
$$

where $\mathbf{A}^{S}, \mathbf{A}^{F}$ are constant complex vector wave amplitudes. With the help of (3.3), (2.6) leads to homogeneous vector equations in $\mathbf{A}^{S}$ and $\mathbf{A}^{F}$. After elimination of $\mathbf{A}^{S}$ and $\mathbf{A}^{F}$, the following quadratic equation in $V^{2}$ is obtained:

$$
\begin{gathered}
\left(V^{2}\right)^{2}+\left[\omega \omega c_{2 F}^{2}+\omega \omega\left(\frac{\bar{\xi}^{S}}{k^{2}}\right)+\omega \omega\left(\frac{\bar{\xi}^{F}}{k^{2}}\right)-c_{2 S}^{2}\right] V^{2} \\
-\left[\omega \omega c_{2 S}^{2} c_{2 F}^{2}+\omega \omega c_{2 S}^{2}\left(\frac{\bar{\xi}^{F}}{k^{2}}\right)+\omega c_{2 F}^{2}\left(\frac{\bar{\xi}^{S}}{k^{2}}\right)\right]=0 .
\end{gathered}
$$

Equation (3.4) gives two roots $V_{4}^{2}, V_{5}^{2}$, which correspond to complex speeds of two dispersive coupled transverse waves in a thermally conducting mixture of elastic solid and Newtonian fluid. Hence, the analytical solution indicates the existence of five dispersive plane waves in two-dimensional model of a thermally conducting mixture of an elastic solid and a Newtonian fluid for both L-S and G-L theories.

\section{Particular Cases}

(i) In absence of fluid, (3.2) reduces to the following quadratic equation:

$$
\tau_{m}^{\prime}\left(V^{2}\right)^{2}-\left[\tau_{m}^{\prime} c_{1 S}^{2}+K_{\tau}\right] V^{2}+c_{1 S}^{2} K_{\tau}=0
$$

which gives complex speeds of two coupled longitudinal waves for both L-S and G-L theories whereas (3.4) reduces to a linear equation which gives the speed of transverse wave.

(ii) In absence of thermal effects, the cubic equation (3.2) reduces to the following quadratic equation:

$$
\begin{aligned}
& \left(V^{2}\right)^{2}+\left[\omega c_{1 F}^{2}-c_{1 S}^{2}+\frac{\omega \omega\left(\bar{\xi}^{S}+\bar{\xi}^{F}\right)}{k^{2}}\right] V^{2} \\
& \quad-\left\{\omega^{2} c_{1 F}^{2}\left(\frac{\bar{\xi}^{S}}{k^{2}}\right)+\omega \omega c_{1 S}^{2} c_{1 F}^{2}+\omega \omega c_{1 S}^{2}\left(\frac{\bar{\xi}^{F}}{k^{2}}\right)\right\}=0 .
\end{aligned}
$$

The roots of (4.2) correspond to the complex phase speeds of two coupled longitudinal waves in a mixture of an elastic solid and a Newtonian fluid. 


\section{Reflection from Traction-Free Thermally Insulated Surface}

We consider the half-space of the medium with $x$-axis along the thermally insulated stressfree surface and $z$-axis into the medium. For incident coupled longitudinal waves or coupled transverse waves, there will be five reflected waves into the medium as shown in Figure 1. The required boundary conditions at stress-free thermally insulated surface are

$$
t_{z z}^{S}=0, \quad t_{z x}^{S}=0, \quad t_{z z}^{F}=0, \quad t_{z x}^{F}=0, \quad \frac{\partial T}{\partial z}=0 .
$$

Here,

$$
\begin{aligned}
& t_{z z}^{S}=\lambda^{S} u_{1,1}^{S}+\left(\lambda^{S}+2 \mu^{S}\right) u_{3,3}^{S}-\beta_{0}\left(T+t_{1} \dot{T}\right), \\
& t_{z x}^{S}=\mu^{S}\left(u_{1,3}^{S}+\dot{u}_{3,1}^{S}\right) \\
& t_{z z}^{F}=\lambda^{F} \dot{u}_{1,1}^{F}+\left(\lambda^{F}+2 \mu^{F}\right) \dot{u}_{3,3}^{F} \\
& t_{z x}^{F}=\mu^{F}\left(\dot{u}_{1,3}^{F}+\dot{u}_{3,1}^{F}\right) .
\end{aligned}
$$

The appropriate potentials required to satisfy the above boundary conditions are

$$
\begin{aligned}
q^{S}= & A_{0} \exp \left[\iota k_{1}\left(\sin \theta_{0} x-\cos \theta_{0} z\right)-\iota \omega_{1} t\right] \\
& +\Sigma\left\{A_{i} \exp \left[\iota k_{i}\left(\sin \theta_{i} x+\cos \theta_{i} z\right)-\iota \omega_{i} t\right]\right\}, \quad(i=1,2,3), \\
q^{F}= & \xi_{1} A_{0} \exp \left[\iota k_{1}\left(\sin \theta_{0} x-\cos \theta_{0} z\right)-\iota \omega_{1} t\right] \\
& +\Sigma\left\{\xi_{i} A_{i} \exp \left[\iota k_{i}\left(\sin \theta_{i} x+\cos \theta_{i} z\right)-\iota \omega_{i} t\right]\right\}, \quad(i=1,2,3), \\
T= & \eta_{1} A_{0} \exp \left[\iota k_{1}\left(\sin \theta_{0} x-\cos \theta_{0} z\right)-\iota \omega_{1} t\right] \\
& +\Sigma\left\{\eta_{i} A_{i} \exp \left[\iota k_{i}\left(\sin \theta_{i} x+\cos \theta_{i} z\right)-\iota \omega_{i} t\right]\right\}, \quad(i=1,2,3), \\
U^{S}= & B_{0} \exp \left[\iota k_{4}\left(\sin \theta_{0} x-\cos \theta_{0} z\right)-\iota \omega_{4} t\right] \\
& +\Sigma\left\{A_{j} \exp \left[\iota k_{j}\left(\sin \theta_{j} x+\cos \theta_{j} z\right)-\iota \omega_{j} t\right]\right\}, \quad(j=4,5), \\
U^{F}= & \eta_{4} B_{0} \exp \left[\iota k_{4}\left(\sin \theta_{0} x-\cos \theta_{0} z\right)-\iota \omega_{4} t\right] \\
& +\Sigma\left\{\eta_{j} A_{j} \exp \left[\iota k_{j}\left(\sin \theta_{j} x+\cos \theta_{j} z\right)-\iota \omega_{j} t\right]\right\}, \quad(j=4,5) .
\end{aligned}
$$

Here $A_{0}, A_{1}, A_{2}, A_{3}, B_{0}, A_{4}$ and $A_{5}$ are the amplitude of incident coupled longitudinal I (CL I), reflected coupled longitudinal I (CL I), reflected coupled longitudinal II (CL II), reflected coupled longitudinal III (CL III), incident coupled transverse I (CT I), reflected coupled transverse I (CT I) and reflected coupled transverse II (CT II) waves, respectively, where 


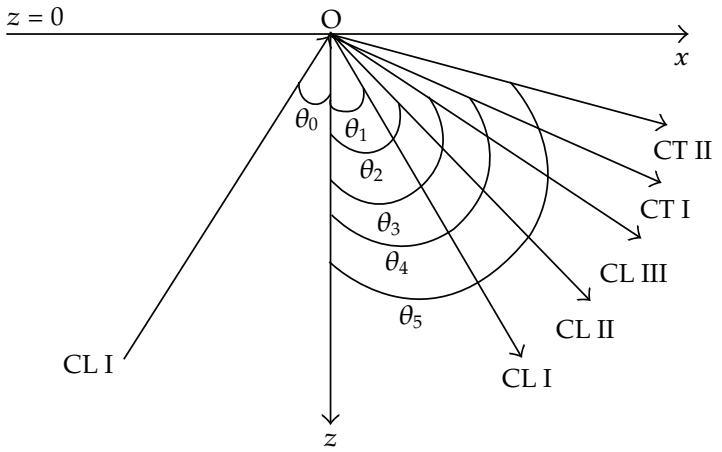

Figure 1: Geometry of the problem showing incident and reflected waves.

$B_{0}=0$ for incident coupled longitudinal I (CL I) wave, $A_{0}=0$ for incident coupled transverse I (CT I) wave, and

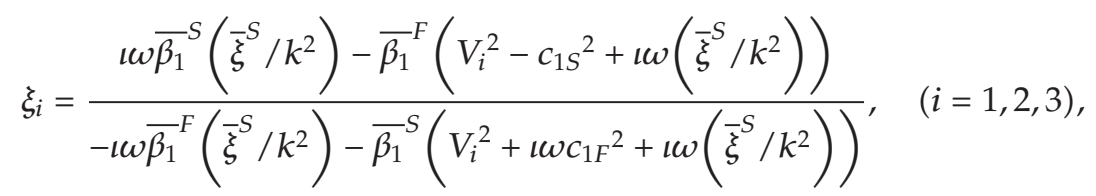

$$
\begin{aligned}
& \eta_{i}=\frac{\iota \omega \bar{\xi}^{F}\left(\bar{s} /\left(1-\omega \tau_{0} \Omega\right)+\bar{\delta}\right)-k^{2}\left(\bar{s} /\left(1-\omega \tau_{0} \Omega\right)-\overline{\beta_{0}}\right)\left(V_{i}^{2}+\iota \omega c_{1 F}{ }^{2}+\omega \omega\left(\bar{\xi}^{F} / k^{2}\right)\right)}{\left(\tau_{m}^{\prime}-\left(\overline{K^{*}} / \tau_{n} V_{i}^{2}\right)\right)\left(V_{i}^{2}+\iota \omega c_{1 F}{ }^{2}+\omega \omega\left(\bar{\xi}^{F} / k^{2}\right)\right)+{\overline{\beta_{1}}}^{F} \tau_{m}\left(\left(\bar{s} /\left(1-\omega \tau_{0} \Omega\right)\right)+\bar{\delta}\right)}, \\
& (i=1,2,3) \text {, } \\
& \eta_{j}=\frac{\iota \omega\left(\bar{\xi}^{F} / k^{2}\right)}{\left(V_{j}^{2}+\iota \omega c_{2 F^{2}}\right)+\iota \omega\left(\bar{\xi}^{F} / k^{2}\right)}, \quad(j=4,5),
\end{aligned}
$$

and the relations between angles of incidence and reflection are given as

$$
\frac{\sin \theta_{0}}{\left(c_{1} \text { or } c_{4}\right)}=\frac{\sin \theta_{1}}{c_{1}}=\frac{\sin \theta_{2}}{c_{2}}=\frac{\sin \theta_{3}}{c_{3}}=\frac{\sin \theta_{4}}{c_{4}}=\frac{\sin \theta_{5}}{c_{5}},
$$

and, $V_{j}^{-1}=c_{j}^{-1}-\imath \omega^{-1} q_{j}$, where $c_{j}$ and $q_{j},(j=1,2, \ldots, 5)$ are real phase speeds and attenuations of coupled longitudinal and transverse waves.

Using the appropriate potentials given by (5.3) with the required boundary conditions (5.1), the following nonhomogeneous system of five equations is obtained

$$
\Sigma a_{i j} Z_{j}=b_{i,} \quad(i, j=1,2, \ldots, 5),
$$

where $a_{i j}, b_{i}$, and $Z_{j}$ are given in Appendix B. 


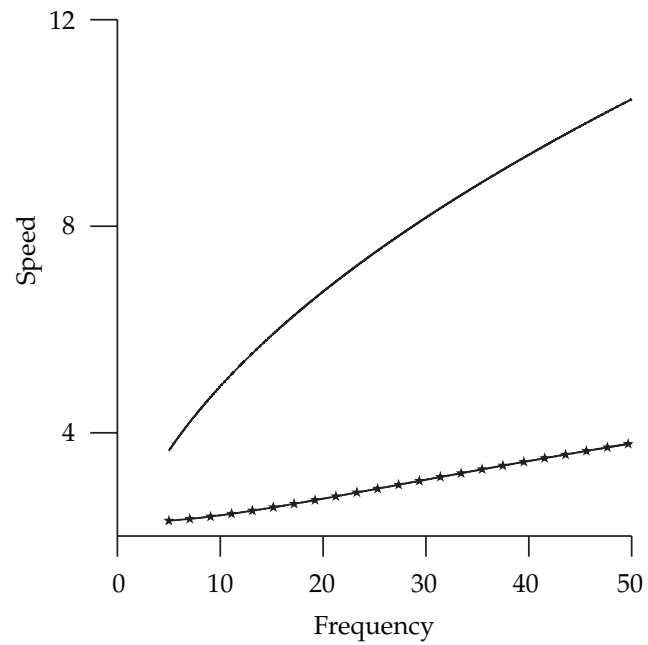

CL I wave

With thermal effects

$\star$ Without thermal effects

(a)

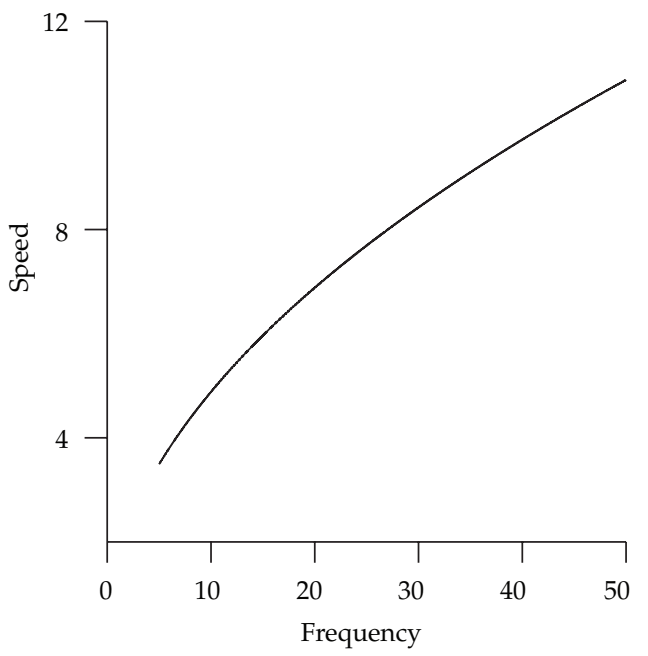

CL III wave

- With thermal effects

(c)

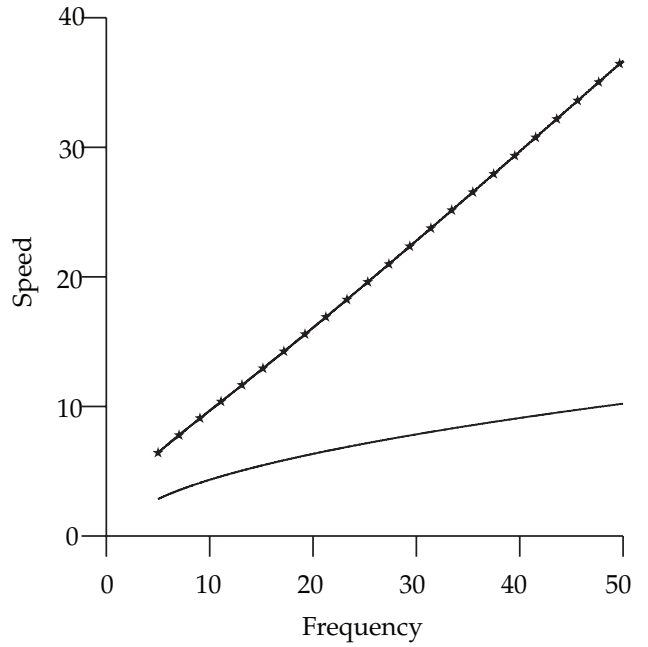

CL II wave

With thermal effects

* Without thermal effects

(b)

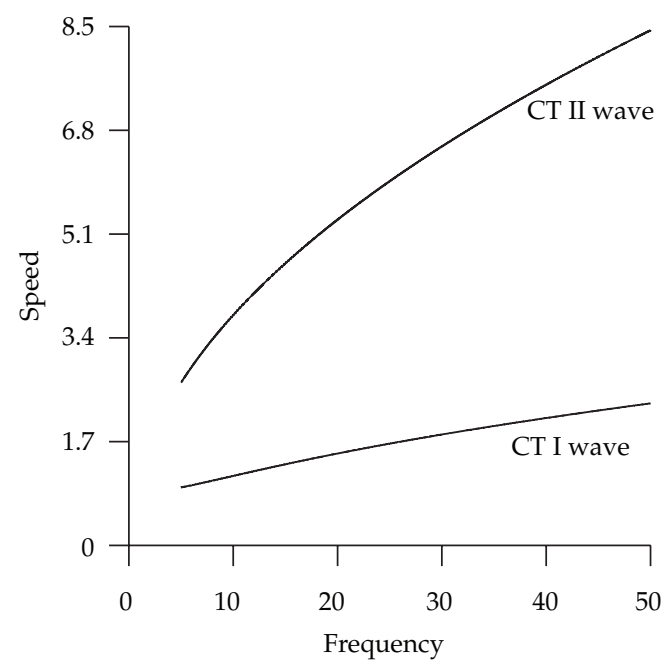

(d)

Figure 2: Thermal effects on speeds of coupled waves against frequency.

\section{Numerical Results and Discussion}

In this section, the complex absolutes of phase speeds and reflection coefficients are computed with the following physical constants at $T_{0}=293^{\circ} \mathrm{K}$,

$$
\begin{aligned}
& \lambda^{S}=7.59 \times 10^{9} \mathrm{~N} \mathrm{~m}^{-2}, \mu^{S}=1.89 \times 10^{9} \mathrm{~N} \mathrm{~m}^{-2}, \\
& \lambda^{F}=2.14 \times 10^{9} \mathrm{~N} \mathrm{~m}^{-2}, \mu^{F}=0.45 \times 10^{9} \mathrm{~N} \mathrm{~m}^{-2}, \\
& \rho^{S}=2192 \mathrm{Kg} \mathrm{m}^{-3}, \rho^{F}=1010 \mathrm{Kg} \mathrm{m}^{-3}, C_{0}=96.3 \mathrm{~J} \mathrm{Kg}^{-1} \mathrm{~K}^{-1},
\end{aligned}
$$




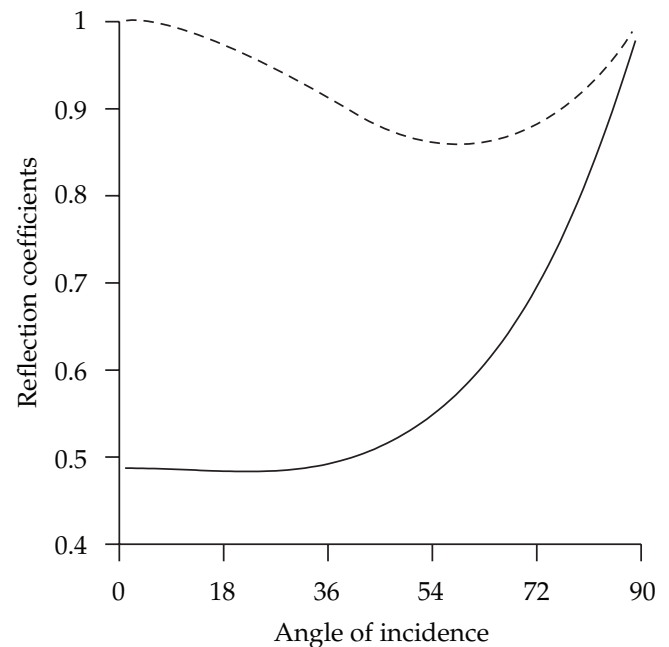

Reflected CL I

With thermal effects

- - Without thermal effects

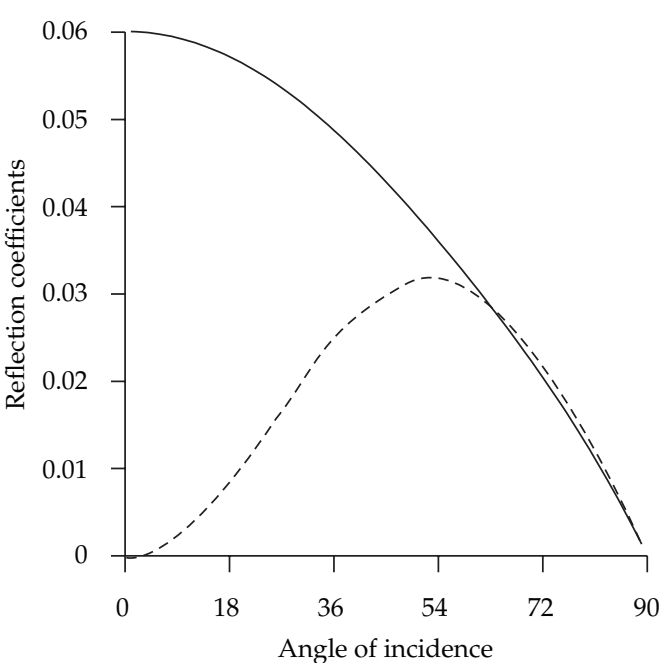

Reflected CL II

With thermal effects

- - Without thermal effects

(a)

(b)

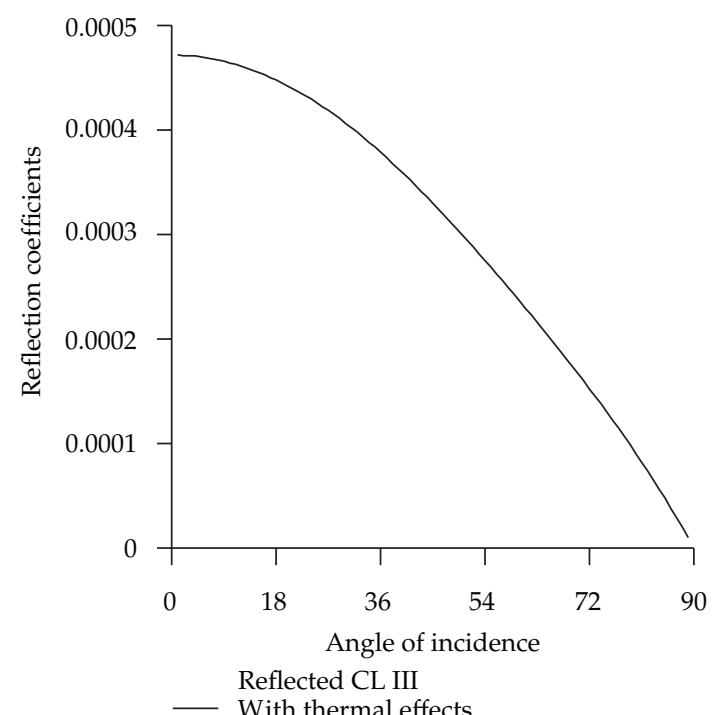

(c)

Figure 3: Thermal effects on coefficients of reflected coupled longitudinal waves for incident CL I wave.

$$
\begin{aligned}
& K^{*}=2.51 \mathrm{~W} \mathrm{~m}^{-1} \mathrm{~K}^{-1}, \mathrm{~s}=0.021 \mathrm{~J}, \xi=750 \mathrm{Kg} \mathrm{m}^{-3} \mathrm{~s}^{-1} \\
& t_{0}=0.005 \mathrm{~s}, t_{1}=0.006 \mathrm{~s}, \beta_{0}=0.0005, \delta=0.0001, n=0.15
\end{aligned}
$$

The densities of solid, fluid, and mixture are related as $\rho=(1-n) \rho^{S}+n \rho^{F}$, where $n$ is porosity of the mixture. 


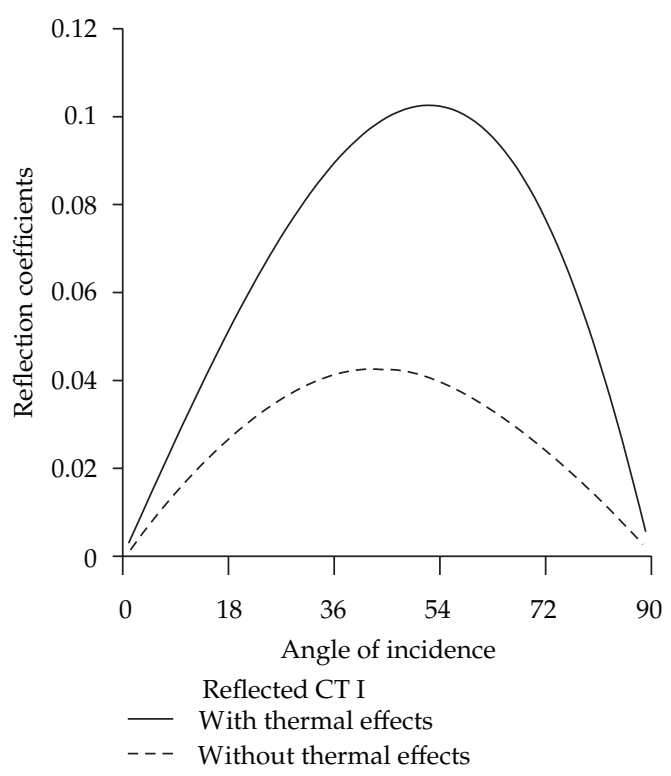

(a)

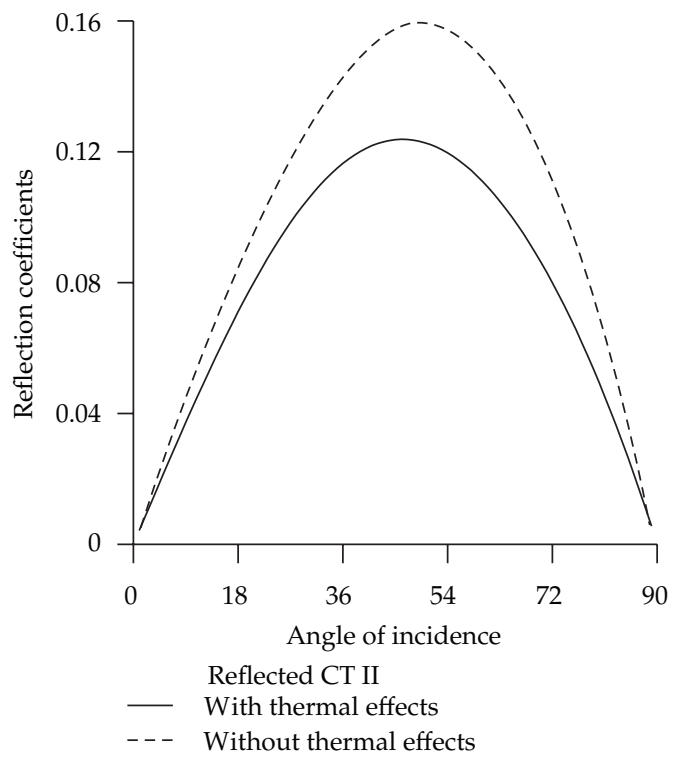

(b)

Figure 4: Thermal effects on coefficients of reflected coupled transverse waves for incident CL I wave.

Numerical computations of phase speeds and reflection coefficients are restricted to the particular case of Lord-Shulman theory only. The phase speeds of the coupled waves are shown graphically in Figure 2 for the range $5 \mathrm{~Hz} \leq \omega \leq 50 \mathrm{~Hz}$ of frequency. It is observed that the phase speeds of all coupled longitudinal waves increase with the increase in frequency. In absence of thermal effects, the phase speed of CL I wave decreases at each value of frequency, whereas the phase speed of CL II increases. The wave CL III will disappear in absence of thermal parameters. The phase speeds of coupled transverse waves (CT I and CT II) remain unaffected by thermal effects.

Reflection coefficients are computed for incident CL I wave only. The reflection coefficients of coupled longitudinal waves are shown graphically in Figure 3 against the angle of incidence. The reflection coefficients of CL I and CL II waves change with the increase in angle of incidence. The comparison of solid and dotted curves shows the effects of thermal parameters on these coefficients. The reflected CL III wave will disappear in absence of thermal effects. The reflection coefficients of CT I and CT II waves also change with the increase in angle of incidence. These waves are also affected by thermal parameters as shown in Figure 4.

\section{Conclusions}

The generalized thermoelasticity of a mixture of an elastic solid and a Newtonian fluid is developed in accordance with Lord-Shulman and Green-Lindsay theories. The solutions of governing equations suggest that there will exist three coupled longitudinal waves and two coupled transverse waves in the present model. From numerical results, it is observed that the phase speeds of coupled waves increase with the increase in frequency. The presence of thermal parameters affects the phase speeds of coupled longitudinal waves only. Reflection coefficients of all reflected coupled waves are affected significantly by thermal disturbances in the material. 


\section{Appendices}

\section{A. Expressions for $A, B$, and $C$}

$$
\begin{gathered}
A=\frac{\left[\omega c_{1 F}^{2} \tau_{m}^{\prime}-c_{1 S}^{2} \tau_{m}^{\prime}+\iota \omega \tau_{m}^{\prime}\left(\left(\bar{\xi}^{S}+\bar{\xi}^{F}\right) / k^{2}\right)-K_{\tau}+a_{2} \bar{\beta}_{1}^{F} \tau_{m}+\left(a_{1}+a_{2}\right) \bar{\beta}_{1}^{S} \tau_{m}\right]}{\tau_{m}^{\prime}} \\
B=\left[-\iota \omega K_{\tau}\left\{c_{1 F}^{2}+\left(\frac{\bar{\xi}^{F}}{k^{2}}\right)\right\}-c_{1 S}^{2}\left\{\omega \omega c_{1 F}^{2} \tau_{m}^{\prime}+\omega \omega\left(\frac{\bar{\xi}^{F}}{k^{2}}\right) \tau_{m}^{\prime}-K_{\tau}+a_{2} \bar{\beta}_{1}^{F} \tau_{m}\right\}\right. \\
\left.+\omega \omega\left(\frac{\bar{\xi}^{S}}{k^{2}}\right)\left\{\omega c_{1 F}^{2} \tau_{m}^{\prime}-K_{\tau}-a_{1} \bar{\beta}_{1}^{F} \tau_{m}\right\}+\omega \omega \bar{\beta}_{1}^{S} \tau_{m}\left\{a_{2} c_{1 F}^{2}+a_{1} c_{1 F}^{2}+a_{1}\left(\frac{\bar{\xi}^{F}}{k^{2}}\right)\right\}\right] / \tau_{m}^{\prime}, \\
C=\frac{\left[\omega c^{2}{ }_{1 S} K_{\tau}\left\{c_{1 F}^{2}+\left(\bar{\xi}^{F} / k^{2}\right)\right\}+\omega^{2} K_{\tau} c_{1 F}^{2}\left(\bar{\xi}^{S} / k^{2}\right)\right]}{\tau_{m}^{\prime}},
\end{gathered}
$$

and, $a_{1}=\bar{s}^{*}-\bar{\beta}_{0}, a_{2}=\bar{s}^{*}+\bar{\delta}, \bar{s}^{*}=\bar{s} /\left(1-\iota \omega t_{0} \Omega\right), K_{\tau}=\overline{K^{*}} /\left(t_{0} \Omega+\iota / \omega\right), \tau_{m}=1-\imath \omega t_{1}$, $\tau_{m}^{\prime}=\left(1-\iota \omega t_{0}\right) /\left(1-\iota \omega t_{0} \Omega\right)$.

B. Expressions for $a_{i j}, b_{i}$, and $Z_{j}$

$$
\begin{aligned}
& a_{1 i}=k_{i}^{2}\left[\lambda^{S}+2 \mu^{S} \cos ^{2} \theta_{i}+\beta_{0}\left(1-\imath \omega_{i} \tau_{1}\right)\left(\frac{\eta_{i}}{k_{i}^{2}}\right)\right], \quad(i=1,2,3), \\
& a_{1 j}=2 k_{j}^{2} \mu^{S} \sin \theta_{j} \cos \theta_{j} \quad(j=4,5), \\
& a_{2 i}=-k_{i}^{2} \sin \theta_{i} \cos \theta_{i}, \quad(i=1,2,3), \\
& a_{2 j}=k_{j}^{2} \cos 2 \theta_{j}, \quad(j=4,5), \\
& a_{3 i}=k_{i}^{2} \omega_{i} \xi_{i}\left(\lambda^{F}+2 \mu^{F} \cos ^{2} \theta_{i}\right), \quad(i=1,2,3), \\
& a_{3 j}=2 k_{j}^{2} \omega_{j} \eta_{j} \mu^{F} \sin \theta_{j} \cos \theta_{j}, \quad(j=4,5), \\
& a_{4 i}=2 k_{i}^{2} \omega_{i} \xi_{i} \sin \theta_{i} \cos \theta_{i}, \quad(i=1,2,3), \\
& a_{4 j}=-k_{j}^{2} \omega_{j} \eta_{j} \cos 2 \theta_{j}, \quad(j=4,5), \\
& a_{5 i}=k_{i} \eta_{i} \cos \theta_{i,} \quad(i=1,2,3), \\
& a_{5 j}=0, \quad(j=4,5),
\end{aligned}
$$


and $(a)$ for incident CL I wave $\left(\theta_{0}=\theta_{1}\right)$,

$$
\begin{aligned}
& b_{1}=-k_{1}^{2}\left[\lambda^{S}+2 \mu^{S} \cos ^{2} \theta_{0}+\beta_{0}\left(1-\imath \omega_{1} \tau_{1}\right)\left(\frac{\eta_{1}}{k_{1}^{2}}\right)\right] \\
& b_{2}=-k_{1}^{2} \sin \theta_{0} \cos \theta_{0}, \\
& b_{3}=-k_{1}^{2} \omega_{1} \xi_{1}\left(\lambda^{F}+2 \mu^{F} \cos ^{2} \theta_{0}\right), \\
& b_{4}=2 k_{1}^{2} \omega_{1} \xi_{1} \sin \theta_{0} \cos \theta_{0}, \\
& b_{5}=k_{1} \eta_{1} \cos \theta_{0}, \\
& Z_{1}=\frac{A_{1}}{A_{0}}, \quad Z_{2}=\frac{A_{2}}{A_{0}}, \quad Z_{3}=\frac{A_{3}}{A_{0}}, \quad Z_{4}=\frac{A_{4}}{A_{0}}, \quad Z_{5}=\frac{A_{5}}{A_{0}}
\end{aligned}
$$

(b) for incident CT I wave $\left(\theta_{0}=\theta_{4}\right)$,

$$
\begin{aligned}
& b_{1}=2 k_{4}^{2} \mu^{S} \sin \theta_{0} \cos \theta_{0} \\
& b_{2}=-k_{4}^{2} \cos 2 \theta_{0} \\
& b_{3}=2 k_{4}^{2} \omega_{4} \eta_{4} \mu^{F} \sin \theta_{0} \cos \theta_{0} \\
& b_{4}=k_{4}^{2} \omega_{4} \eta_{4} \cos 2 \theta_{0}, \\
& b_{5}=0, \\
& Z_{1}=\frac{A_{1}}{B_{0}}, \quad Z_{2}=\frac{A_{2}}{B_{0}}, \quad Z_{3}=\frac{A_{3}}{B_{0}}, \quad Z_{4}=\frac{A_{4}}{B_{0}}, \quad Z_{5}=\frac{A_{5}}{B_{0}} .
\end{aligned}
$$

Here, the amplitude ratios $Z_{1}, Z_{2}, Z_{3}, Z_{4}$, and $Z_{5}$ correspond to reflected CL I wave, CL II wave, CL III wave, CT I wave, and CT II wave, respectively.

\section{References}

[1] C. Truesdell, "Mechanical basis of diffusion," The Journal of Chemical Physics, vol. 37, pp. 2336-2344, 1962.

[2] C. Truesdell and R. A. Toupin, "The classical field theories," in Encyclopedia of Physics, S. Flugge, Ed., vol. III/I, pp. 226-793, Springer, Berlin, Germany, 1960.

[3] A. E. Green and P. M. Naghdi, "On basic equations for mixtures," The Quarterly Journal of Mechanics and Applied Mathematics, vol. 22, pp. 427-438, 1969.

[4] R. J. Atkin and R. E. Craine, "Continuum theories of mixtures: basic theory and historical development," The Quarterly Journal of Mechanics and Applied Mathematics, vol. 29, no. 2, pp. 209-244, 1976.

[5] R. M. Bowen, "Theory of mixtures," in Continuum Physics, A. C. Eringen, Ed., vol. 3, pp. 1-317, Academic Press, New York, NY, USA, 1976.

[6] A. Bedford and D. S. Drumheller, "Theories of immiscible and structured mixtures," International Journal of Engineering Science, vol. 21, no. 8, pp. 863-960, 1983.

[7] I. Muller, "A thermoelastic theory of mixture of fluids," Archive for Rational Mechanics and Analysis, vol. 43, pp. 45-61, 1971. 
[8] N. T. Dunwoody, "A thermomechanical theory of diffusion in solid-fluid mixtures," Archive for Rational Mechanics and Analysis, vol. 38, no. 5, pp. 348-371, 1970.

[9] S. Krishnaswamy and R.C. Batra, "A thermomechanical theory od solid-fluid mixtures," Mathematics and Mechanics of Solids, vol. 2, pp. 143-151, 1997.

[10] D. Iesan, "On the theory of mixtures of elastic solids," Journal of Elasticity, vol. 35, no. 1-3, pp. 251-268, 1994.

[11] D. Iesan, "A theory of mixtures with different constituent temperatures," Journal of Thermal Stresses, vol. 20, no. 2, pp. 147-167, 1997.

[12] D. Iesan, "On the theory of viscoelastic mixtures," Journal of Thermal Stresses, vol. 27, no. 12, pp. 11251148, 2004.

[13] K. R. Rajagopal and L. Tao, Mechanics of Mixtures, vol. 35 of Series on Advances in Mathematics for Applied Sciences, World Scientific, River Edge, NJ, USA, 1995.

[14] A. Pompei and A. Scalia, "On the dynamic theory of mixtures of thermoelastic solids," Journal of Thermal Stresses, vol. 22, no. 1, pp. 23-34, 1999.

[15] A. C. Eringen, "Micropolar mixture theory of porous media," Journal of Applied Physics, vol. 94, pp. 4184-4190, 2003.

[16] H. Lord and Y. Shulman, "A generalized dynamical theory of thermoelasticity," Journal of the Mechanics and Physics of Solids, vol. 15, pp. 299-309, 1967.

[17] A. E. Green and K. A. Lindsay, "Thermoelasticity," Journal of Elasticity, vol. 2, pp. 1-7, 1972. 


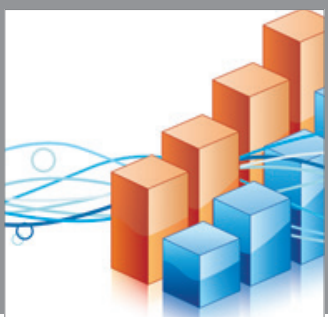

Advances in

Operations Research

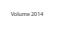

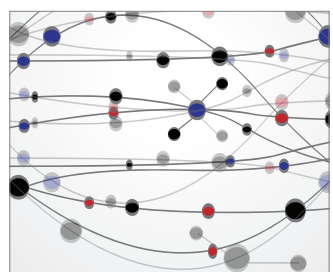

\section{The Scientific} World Journal
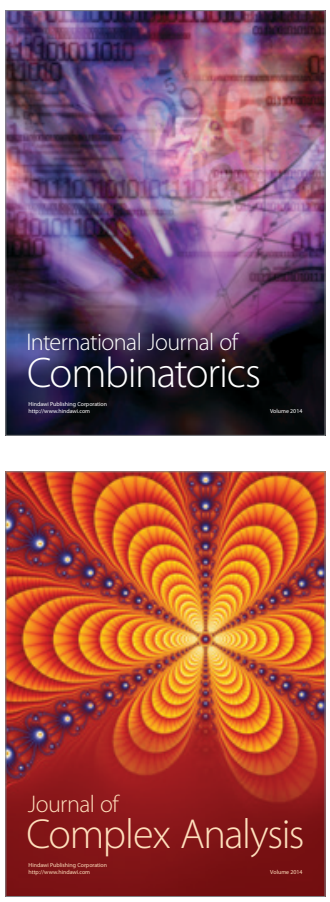

International Journal of

Mathematics and

Mathematical

Sciences
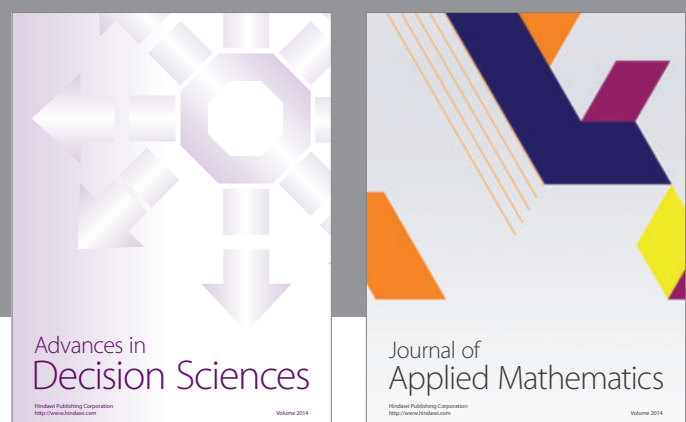

Journal of

Applied Mathematics
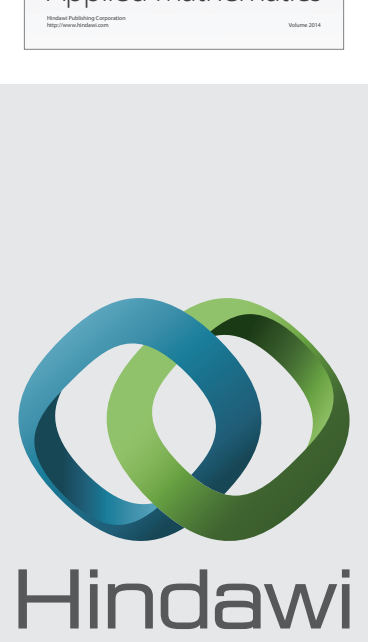

Submit your manuscripts at http://www.hindawi.com
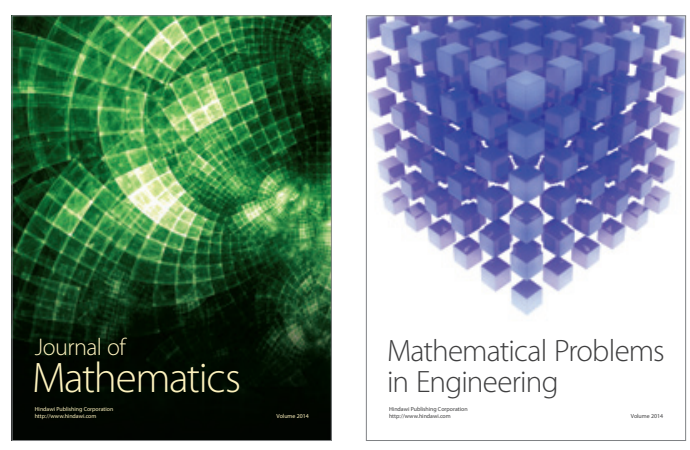

Mathematical Problems in Engineering
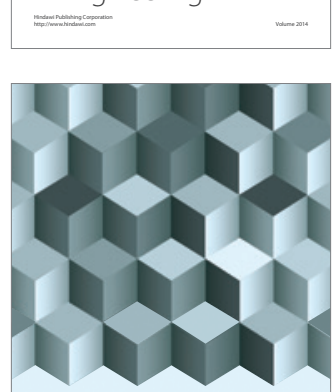

Journal of

Function Spaces
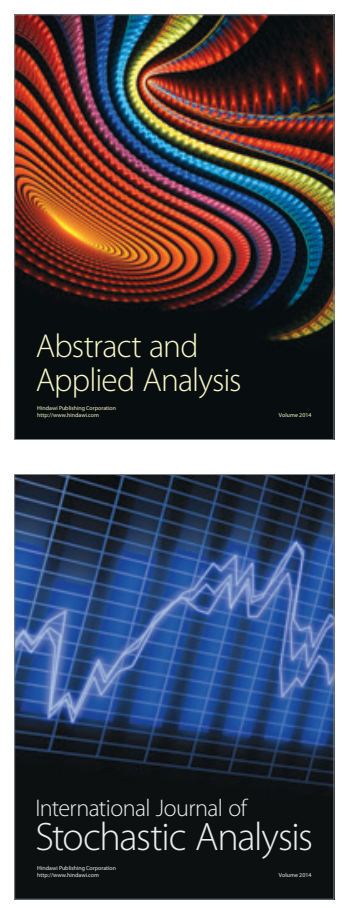

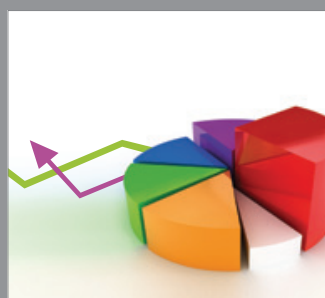

ournal of

Probability and Statistics

Promensencen
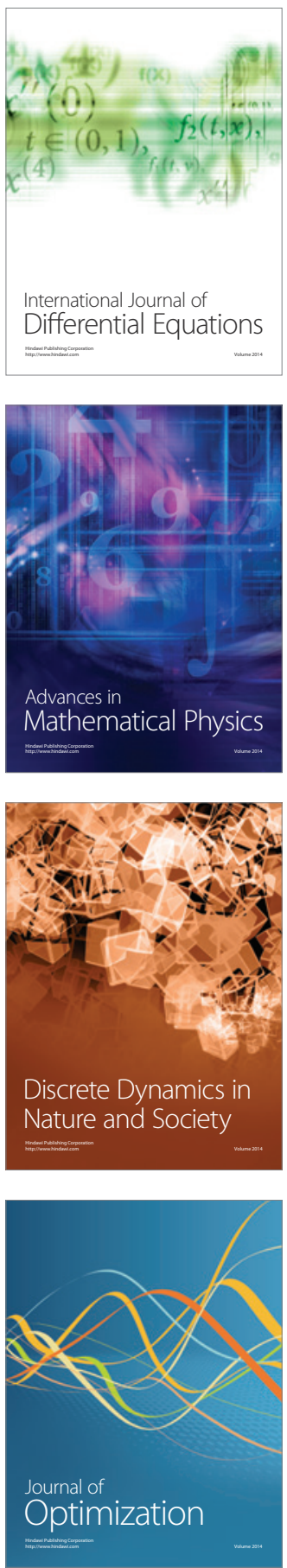